\title{
Hybrid Methodology for Image Segmentation Based on Active Contour Module and Alpha-Shape Theory
}

\author{
Mohammed Sabbih Hamoud Al-Tamimi ${ }^{1}$ \\ ${ }^{1}$ Department of Computer Science, College of Science, University of Baghdad, Iraq \\ Correspondence: Mohammed Sabbih Hamoud Al-Tamimi, Department of Computer Science, College of Science, \\ University of Baghdad, Baghdad, Iraq. Tel: 964-771-3066-766. E-mail: m_altamimi75@yahoo.com
}

Received: June 22, 2016

doi:10.5539/mas.v10n11p30
Accepted: July 4, 2016

Online Published: July 17, 2016

URL: http://dx.doi.org/10.5539/mas.v10n11p30

\begin{abstract}
The concept of the active contour model has been extensively utilized in the segmentation and analysis of images. This technology has been effectively employed in identifying the contours in object recognition, computer graphics and vision, biomedical processing of images that is normal images or medical images such as Magnetic Resonance Images (MRI), X-rays, plus Ultrasound imaging. Three colleagues, Kass, Witkin and Terzopoulos developed this energy, lessening "Active Contour Models" (equally identified as Snake) back in 1987. Being curved in nature, snakes are characterized in an image field and are capable of being set in motion by external and internal forces within image data and the curve itself in that order. The present study proposes the use of a hybrid image segmentation technique to acquire precise segmentation outcomes, while engaging "Alpha Shape ( $\alpha$-Shape)" in supposition to derive the original contour, followed by a refining process through engaging a conventional active contour model. Empirical results show high potential in the suggested computational method. Trials indicate that the primary contour is capable of being precisely set next to the objective contour and effectively have these objective contours extracted, devoid of any contour instigation. Some of the benefits associated with the novel hybrid contour include minimized cost of computation, enhanced anti-jamming capability, as well as enlarged utilization array of snake model.
\end{abstract}

Keywords: active contours models, Alpha Shape, automatic initialization, image segmentation, snake

\section{Introduction}

By definition, segmentation of images entails the identification and grouping together of features or regions sharing identical qualities. Segmentation exploits arithmetical categorization, threshold, detection of edges and regions, or a mixture of these approaches to resolve segmentation problems. These approaches range from edge-based, connectivity-based, threshold-based, to region-based (Pichumani, 1997). Region-based methods are dependent on general patterns in concentration values within a group of adjacent pixels. This group or cluster is identified as the region, while the objective of the segmentation technique mirrors grouping of regions, irrespective of their anatomical or functional roles. Threshold-based approach is highly dependent on the local pixel information. Its competence is witnessed when the object's intensity levels fall evenly outside the array of background levels. With the image's spatial information being overlooked, problems became evident at region boundaries that were blurred. Edge-based approaches are highly dependent on disruption in image values between different regions, while the purpose of the segmentation operation is to precisely identify the margin demarcating these regions. As for the connectivity-based approach, it is highly dependent on a curve identified as active contour created by various positions on the image. Deformable models (Terzopoulos, Witkin, \& Kass, 1988), or active models (Kass, Witkin, \& Terzopoulos, 1987), have been extensively employed in image segmentation (Gastaud, Fellow, Aubert, \& Ý, 2004; Haud \& Montanvert, 1999; McInerney \& Terzopoulos, 1999; T. McInerney \& Terzopoulos, 1995) as well as tracking of objects (Mansouri, Mukherjee, Member, \& Acton, 2004; Paragios \& Deriche, 2000; Ray, Acton, \& Ley, 2002; Ray \& Acton, 2004). Regardless of the various prevailing customized types of active models (Ootes, Aylor, Ooper, \& Graham, 1995), the most extensively utilized models are inclusive of active surfaces and active contours (Kass et al., 1987; Terzopoulos et al., 1988). Collapsing of the active models occurs on the image domain to gain a preferred aspect by decreasing the energy operational subject to certain constrictions. The energy functional normally entails two conditions: an external energy that draws the elastic model to the interest features, as well as an internal energy model responsible for 
limiting the model's rigidity and smoothness.

Back in 1987, Kass introduced active contour models identified as "Energy-Minimizing Curves" or "Snakes" (Kass et al., 1987). Nonetheless, there exist a number of considerations associated with it. To begin with, it is unable to detect high curvature edges in addition to an image's multi-objects. In addition, the position of the primary contour is needed to be adjacent to the edges; if not, there is a likelihood of arriving at erroneous results hence becoming a difficult undertaking. It was in 1997 that another approach was invented by Xu Chenyang and Prince, identified as Gradient Vector Flow (GVF) capable of broadening the dimension of the primary contour setting by efficiently broadening the GVF capturing force, hence being able to arrive to the U-shaped edges (Prince, 1997; Xu \& Prince, 1998). This brings a solution to the traditional snake model's incapacity to converge to concavity, while the problems of the location of primary contours being next to the true edges. Nonetheless, active contour's capturing array is dependent on the level of GVF force field, normally arrived at by the quantity of GVF iterations. There exist a direct proportion between the number of GVF and computational cost, where several GVF iterations results in high computational cost. While computing for convergence, the active contour may be distant from the ideal edges, hence requiring additional convergence iterations. Regrettably, GVF force field entails points of local least amount of energy holding back the convergence of the initial contour.

Another fresh technique of contour, the commencement of GVF snake model was invented by Caixia L. (Caixia, Yanbin, \& Houjun, 2006). Primarily, a theory of "Effective Area" was considered while "Multi-Scale Edge Detection" technique founded on wavelet transform was employed in acquiring edge points. Eventually, a connection of the edge positions on the interested region's outer layer and incessant initial contour was done. "Effective Area" is the set initial contour, in addition to being adjacent to the true contour, capable of extracting the objective contour besides minimizing the computational costs. Nonetheless, this technique also entails some setbacks. Primarily, it is only capable of exploring the edge points onward and it is unable to get rid of the hindrance caused by the burr noise. Additionally, the searching area of this method is uneven and it equally diverges from the bearing of ideal edges while identifying edge points. In the present study, we investigated an enhanced contour initialization procedure characterized with reversal of all benefits achieved in GVF Snake model and offered a solution to the two setbacks in the technique suggested by (Haud \& Montanvert, 1999). Empirical trials reveal that the present approach is characterized with firm resistivity to noise, for instance, fake contours and strings, minimizing the time intricacy, effectively extracting intricate shapes and widening the utilization array greatly.

\section{Original Snake Active Contour Model}

\subsection{Definition}

Kass Witkin and Terzopoulos derived the inventive snake in 1987 (Kass et al., 1987). The term "Snake" was founded on the model's behavior on an image. As a way of lessening its energy, it slides on an image.

$$
\mathrm{v}(\mathrm{s})=[\mathrm{x}(\mathrm{s}), \mathrm{y}(\mathrm{s})] \quad \mathrm{s} \in[0,1]
$$

As evident Equation (1) expresses a snake as a planar parametric curve. The considerations entail snake control points identified as "Snaxels". Such Snaxels are connected jointly to create an active contour as indicated in Figure 1 below. Arguably, the snake as a method cannot automatically be able to detect the margin of the anticipated object in an image. It needs suitable constraints, settings and primary locations of the "Snaxels" irrespective of the concerned margin. On such grounds, some prevailing information regarding the image under assessment is needed for the top level system.

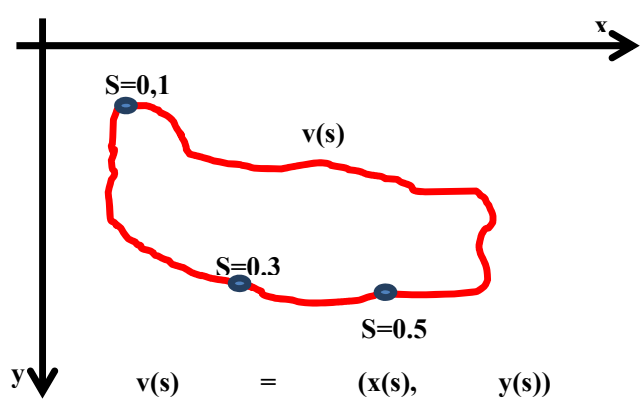

Figure 1. Parametric Curve (Al-Tamimi \& Sulong, 2014) 
Snake's energy function has been represented in Equation (1). With the snake being delineated as an energy decrement spline (Kass et al., 1987), it is able to achieve this by deforming itself, hence, having its energy minimized. The functionality of the energy is in a manner such that the snake arrives at the target's margin. Its behavior is identical to that of a rubber band situated outside an object, hence shirking to arrive at the target's boundary. The initial two phrases of the of snake's energy function stipulated in Equation (2) are identified as "Internal Energy". It is these forces that control the strain and inflexibility of snake model. External Energy is the other term of snake's energy function. It is a derivative of the image and it draws the snake to the target contour.

$$
\mathrm{E}_{\text {snake }}=\int_{0}^{1}\left(\alpha \cdot \mathrm{E}_{\text {elastic }}(\mathrm{v}(\mathrm{s}))+\beta \cdot \mathrm{E}_{\text {bending }}(\mathrm{v}(\mathrm{s}))+\gamma \cdot \mathrm{E}_{\text {image }}(\mathrm{v}(\mathrm{s}))\right) \mathrm{ds}
$$

\subsection{Internal Energy}

The subcomponents of the internal energy include "Bending Force" and "Elasticity Force". Therefore, internal energy is delineated as the summation of such forces with the energy function being articulated as:

$$
\mathrm{E}_{\text {interal }}=\left[\alpha(\mathrm{s})\left|\frac{\mathrm{dv}(\mathrm{s})}{\mathrm{ds}}\right|^{2}+\beta(\mathrm{s})\left|\frac{\mathrm{dv}(\mathrm{s})^{2}}{\mathrm{ds^{2 }}}\right|^{2}\right] / 2
$$

Both the two contour's derivatives (first and second) delineate these energy terms and identified as "Elasticity Force" and "Bending Force", in that order. The snake's tension is controlled by the elastic force. It is able to minimize stretching of the active contour as well as being liable to shrink the contour (as shown in the red arrow of Figure 2 below). An excellent delineation of Bending Force has been termed as the "bending energy makes snake acts like a thin plate" (Kass et al., 1987). It is responsible to control the snake's rigidity. However, its control is limited to the curvature only, hence, excluding the contour's length. At its deformation procedure, a smooth curve or a straight line is maintained (Blue-dot arrow in Figure 2).

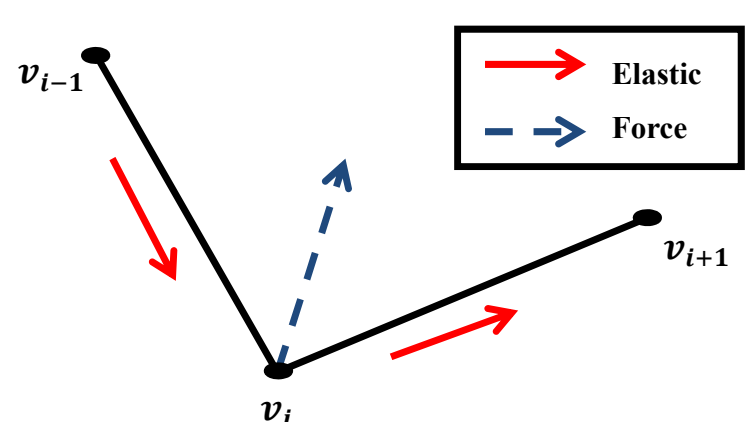

Figure 2. Internal energy (Al-Tamimi \& Sulong, 2014)

Both $\alpha(\mathrm{s})$ and $\beta(\mathrm{s})$ parameters before each term delineates weighing functions. Generally, the weighing functions' values are constants for all snaxels. Considering an opposite set of such constants generates one of the setbacks in snake. These setbacks exhibit huge impacts on the behavior of the snake in addition to being totally in control of deformation process' performance. Every object within an image needs a different set of constant value in order to achieve excellent performance from the snake. One sure way of achieving a solution to this setback has enable the snake to vigorously alter these values to proper values within the process of deformation. Nonetheless, it is through a computer that topologies or shapes of an image object are automatically detected. Therefore, the way out is to consider future improvements of the snake. At present, these constraints are expected to be selected by the users during the initialization phase.

\subsection{External Energy}

The image data is employed in deriving external energy. The snake is drawn towards the target contour by the image-compelled force. The equations below aids in the definition of the energy term (Kass et al., 1987).

$$
\begin{gathered}
\mathrm{E}_{\text {external }}(\mathrm{s})=-\gamma(\mathrm{s}) \cdot|\nabla(\mathrm{I}(\mathrm{s}))|^{2} \\
\mathrm{E}_{\text {external }}(\mathrm{s})=-\gamma(\mathrm{s}) \cdot\left|\nabla\left(\mathrm{G}_{\sigma}(\mathrm{s}) * \mathrm{I}(\mathrm{s})\right)\right|^{2}
\end{gathered}
$$


$\nabla$ Denotes gradient operator while I(s) delineates the image's intensity at s. G(s), $\sigma$ is Two-Dimessional (2D) Gaussian functions having a standard deviation of $\sigma$. Nonetheless, image force control is achieved by engaging the weighing function $\gamma(s)$. The snake's capture range is increased by the application of Gaussian filter to the inventive image. Blurring in images is an outcome of the application of Gaussian filters. The figure below (Figure 3) demonstrates a typical illustration of a blurring effect of an edge. The diffusion of the image force is from one edge (blue-dot line) to another edge (red lines). This result in the widening of the snake's capture array.

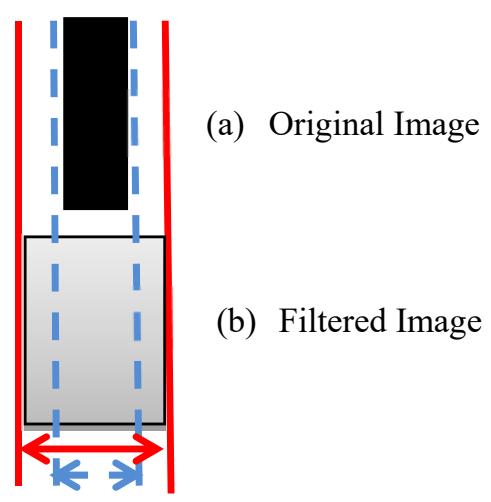

Figure 3. Image Force (Al-Tamimi \& Sulong, 2014)

Normally, Equation (5) is employed in calculating the external force, given that the square of the gradient enormity of concentration itself entails a lesser capture array. In addition, the snake's capture array is highly dependent on the Standard Deviation (SD) $\sigma$. The outcome of larger SD is that the objects' margins tend to become blurry. This is occasionally vital in aiding an active contour to get drawn towards the anticipated boundary. In order to have snake's external force and GVF's sensitivity of the nose is evaluated (Xu \& Prince, 2006), we incorporate impulse noise to the images and evaluate their GVF energy with the inventive image, hence endeavoring to realize the extent unto which noise can impact the GVF value as well as the active contour fragmentation algorithm. The nine images utilized have been illustrated in Table 1 together with their GVF energies as well as GVF energy computed from "Salt and Pepper Noise" and "Gaussian Noise".

Table 1. GVF energy calculated from nine original images and their "Salt and Pepper Noise" and "Gaussian Noise" images (Continue ...)

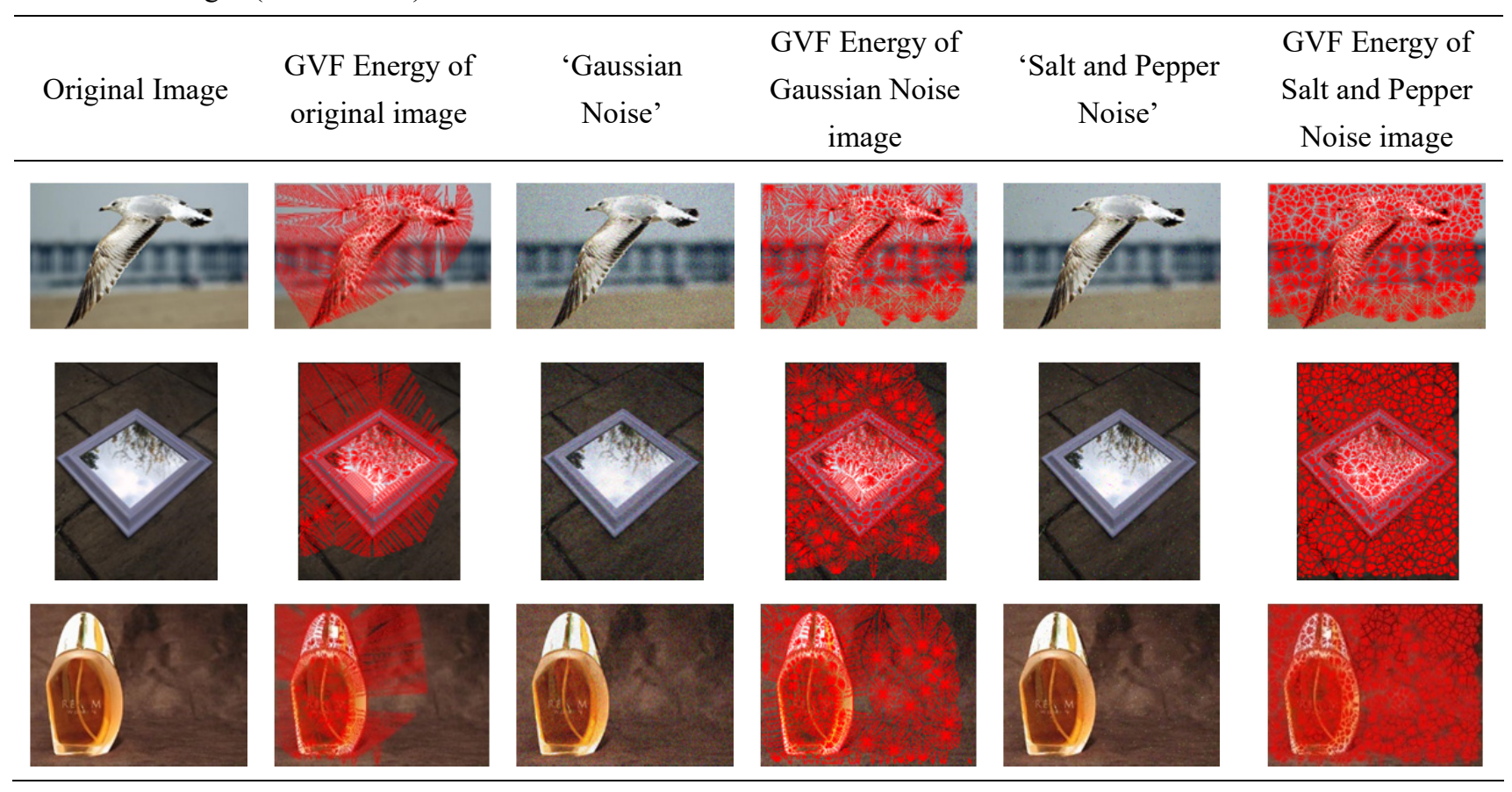


Table 2. GVF energy calculated from nine original images and their "Salt and Pepper Noise" and "Gaussian Noise" images.

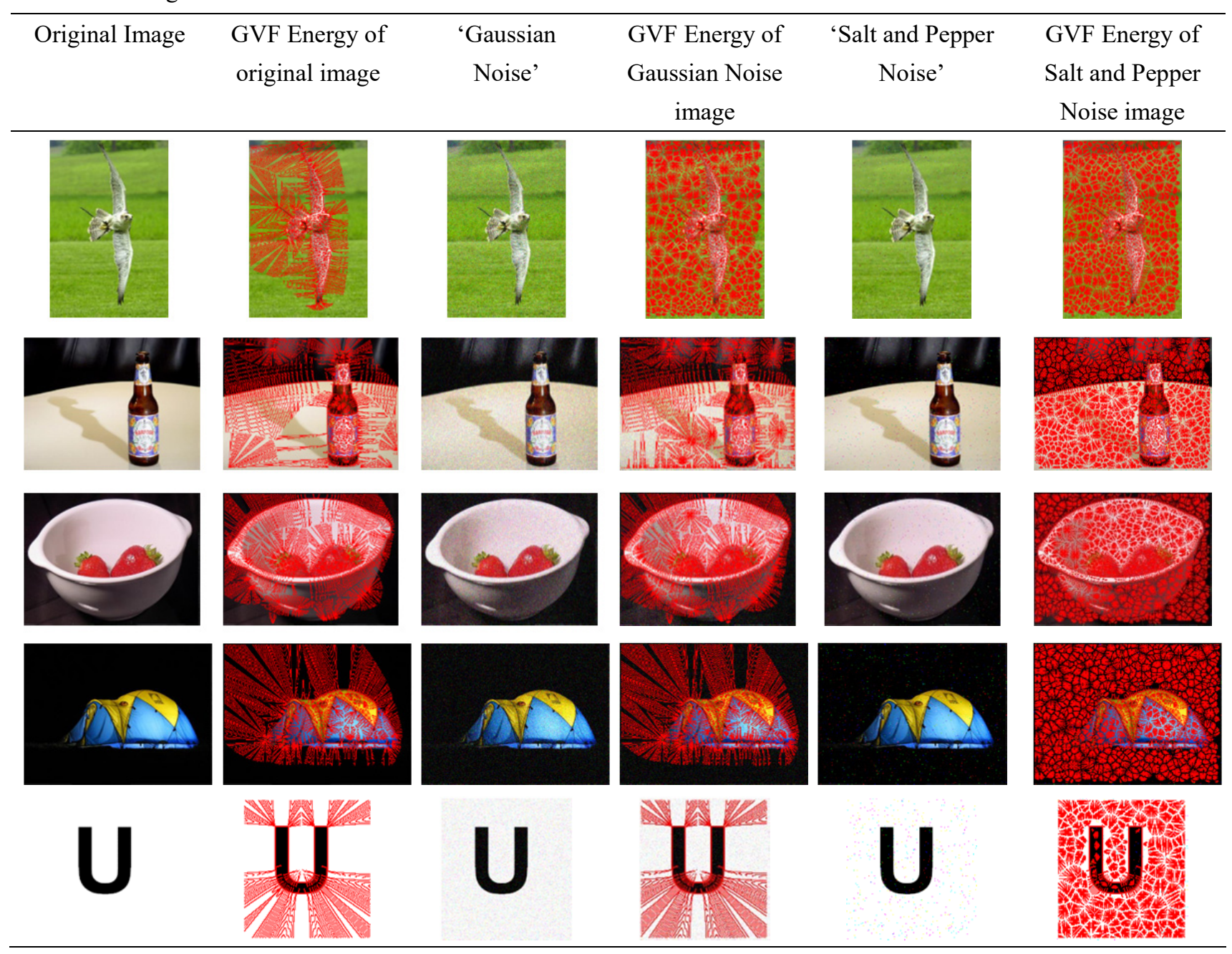

As shown in Table 1, the GVF snake offers excellent outcomes considering original images. However, becomes unable to arrive at the shape plus the object since it faces distractions from local impulse noise in "Salt and Pepper Noise" and "Gaussian Noise" images.

\subsection{Snakes Algorithms}

Several of the algorithms associated with active contour models entail the following three stages:

a) Initialization Process: for the commencement process, the initial snaxels' locations around the target -object's boundary is set by the user. Concurrently, the considered set of weighing constraints includes $\alpha$, $\beta$, and $\gamma$. Subsequently, it begins to deform itself towards the ideal margins of the object. It is vital that the first contour becomes situated adjacent to the subject boundary owing to the condition that the snake was capable of shifting towards the noises among other unwanted lines or edges on an image upon being situated at a distance from the ideal boundary.

Table 3 exemplifies the outcome of the inventive implementation of the active contour algorithm; three diverse initial contour assessments are depicted with their eventual smoothing to exemplify the significance of the snake's initial contour. 
Table 3. Active contour segmentation result using three different initial contours (represented by red lines with white points)

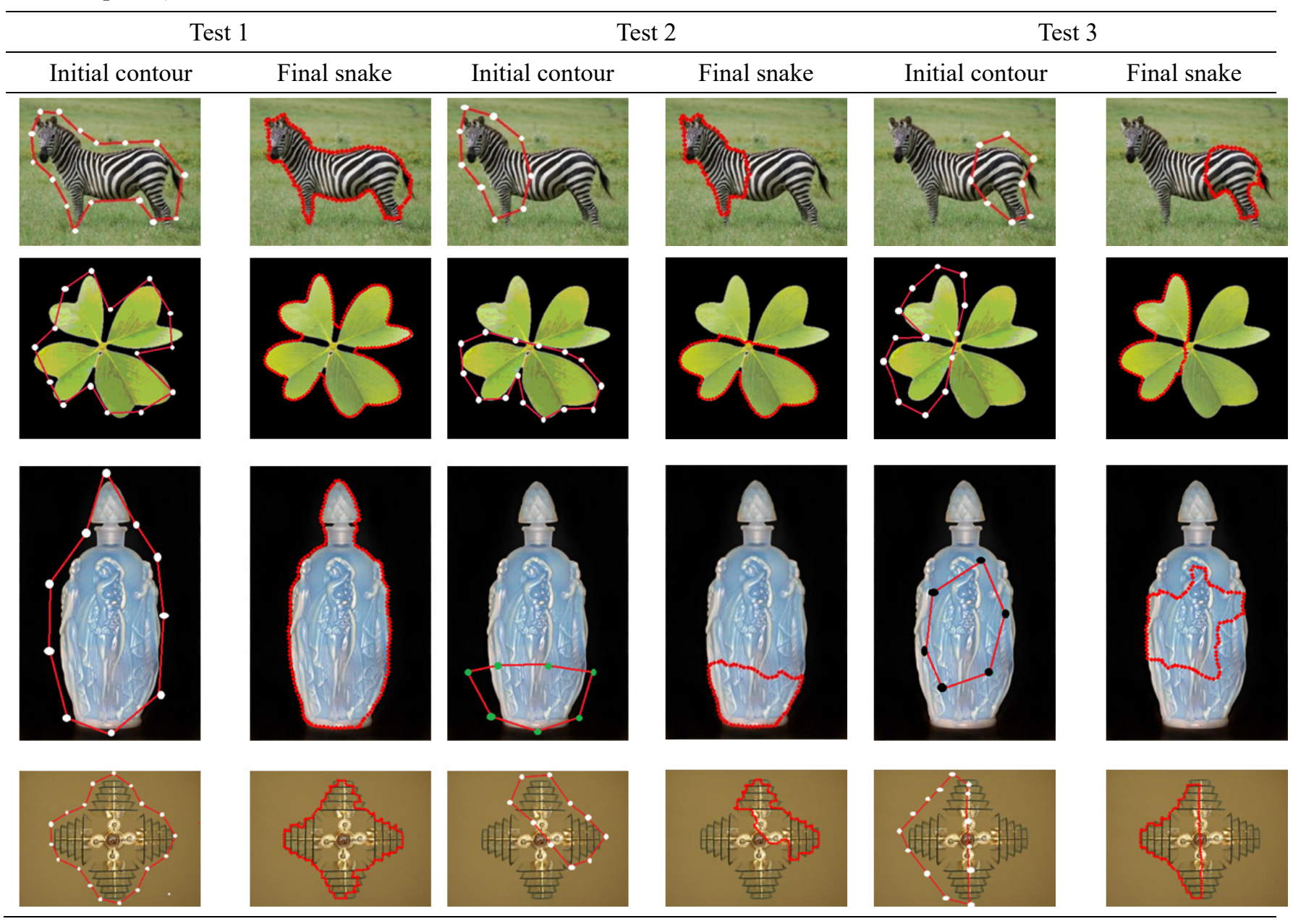

b) Deformation Process: at the deformation phase and procedure, the technique is able to deform itself as an approach of lessening the total energy terms stipulated in Equation 2, for the current process' iteration; a fresh location is searched among adjacent pixels in each snaxel. There are two options of snaxel, to either move towards a low-energy pixel or remain within the same location in case there exist none. There exist several methods to gauge new snaxels' locations.

Among the approaches employed in the realization of a fresh location for each snaxel is the "Greedy Algorithm", perceived being highly simple and unproblematic to be executed. At the iteration phase, the amount of external and internal energies is calculated at a snaxel as well as its adjacent 8 pixels.

The new location is arrived at by considering one with the least energy. For such reasons, the snaxel has the option of shifting to one of the 8 probable adjacent pixels; the red arrows illustrated in Figure 4 below, or equally remain in the same position if none of the energy is smaller compared to the current energy contained by the snaxel.

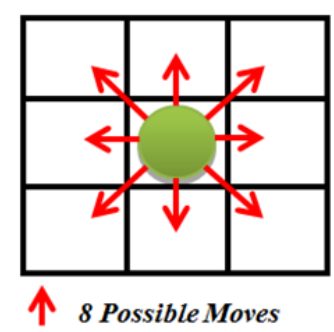

Figure 4. Greedy Algorithm for deformation process of active contour (Al-Tamimi \& Sulong, 2014). 
For the purposes of assessing the competence of GVF snake energy within the active contour's deformation technique, we experimented on the original snake by engaging two diverse images' impulse noises with the outcomes being assessed against those of the original images. Table 3 shows 6 noisy images degraded with a "Salt and Pepper Noise" and "Gaussian Noise" and experimented by use of the inventive snake algorithm.

Table 4. Six images segmented by original active contours using GVF energy model compare with images corrupted with a "Salt and Pepper Noise" and "Gaussian Noise".

\begin{tabular}{ccccc}
\hline \multicolumn{2}{c}{ Original image } & Images corrupted by 'Gaussian noise' & \multicolumn{2}{c}{ Images corrupted by 'Salt and Pepper } \\
Initial contour & Final snake & Initial contour & Final snake & Initial contour \\
\hline
\end{tabular}
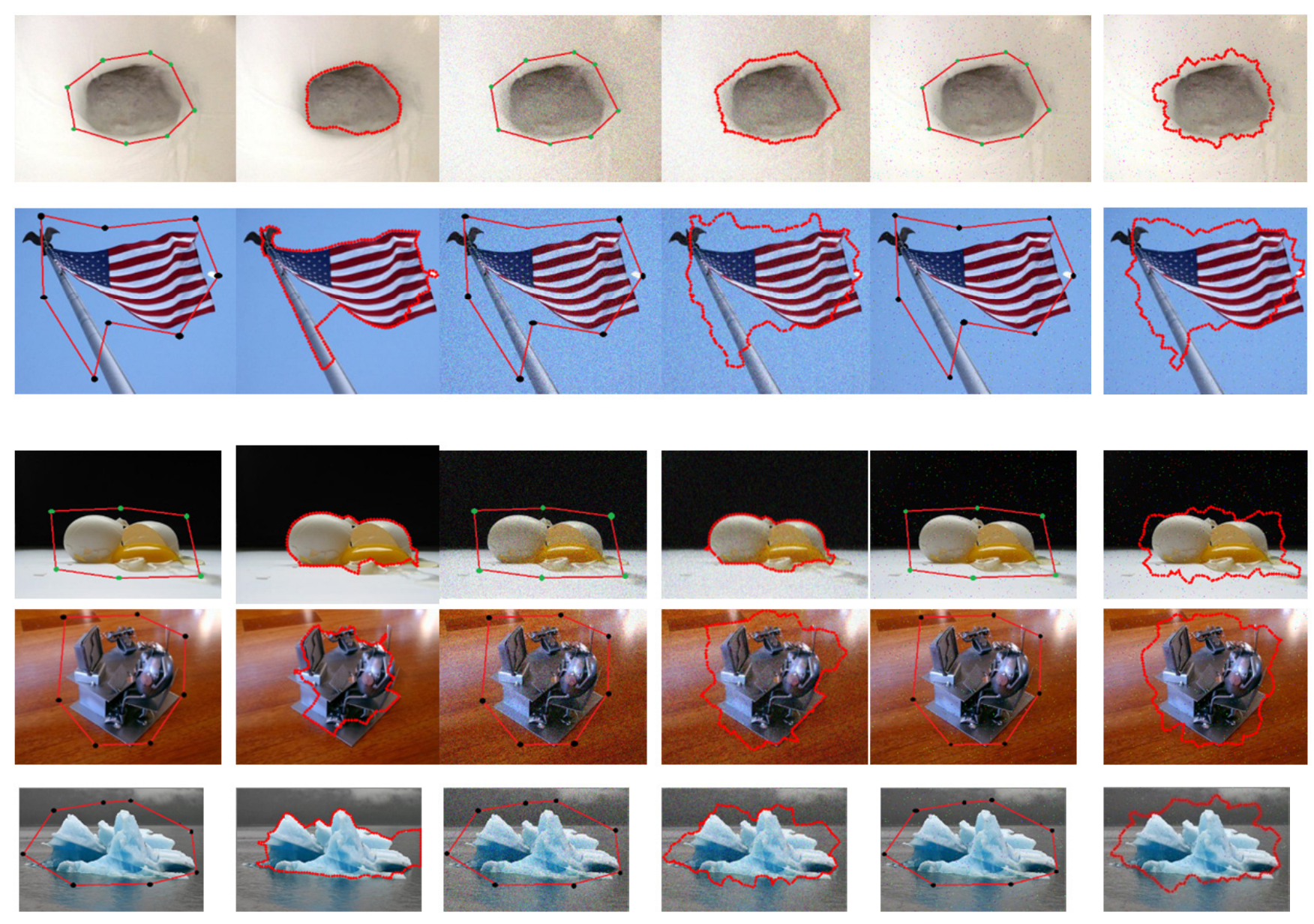

The process of deforming goes on up to a period when the snake is trapped in one of the lapsing techniques, the subsequent section offers a discussion of the termination processes. 
C) Termination of the Snake: at some time instance, there is the need to impede the snake's deformation process.

As expected, the snake will end its deformation where the snaxels are unable to identify fresh locations in the adjacent pixels, or basically by arriving at zero and vanishes from the eight possible moves. Nonetheless, it has the option of shifting into a perpetuity loop upon oscillation or relocation along the margin by the snaxels. Based on this argument, there is the need to put in place a given technique for snake's termination. Setting a threshold on the utmost iteration quantity implemented in the deformation technique should be considered as the simplest approach.

This is an assurance that the snake is ceased and it does not shift into an infinity loop. Although it is important to note that it is highly important that the user sets the correct amount of iterations before commencing the deformation procedure. Due to the fact that the rate of iterations is variant and dependent on the object's size and shape, it is highly intricate to approximate the number of iterations required to identify the subject contour. Due to this, termination of the snake would take place prior to its destination or close to the true margin upon setting the least number of utmost iterations. Another technique is by having the threshold set respective of the rate of snaxels shifted among the iterations. For example, having the threshold set at 90 percent of the snaxels' summation would be a typical illustration. This means that the termination of deformation by the snake will occur when 90 percent of snaxels are unmoved. By so doing, the threshold is independent of the size and shape of the target object. Subsequently, the user is not expected to alter the iteration quantity on every target object.

Nonetheless, none of the terminating methods would be valuable since it is, at times, witnessed that the snaxels tend to move along the boundary, while the contour simply shifts very slightly (Choi, Lam, Siu, \& Ic, 2001). For the purposes of prevailing over this setback, different approaches of automated implementations have been suggested. These initialization approaches have been engaged for diverse forms of images such as MR images, human face, as well as the heart's ultrasound images (Codon \& Fristot, 1999; Jia Liang, Ding, \& Wu, 2008; Pluempitiwiriyawej \& Sotthivirat, 2005; Tauber, Batatia, \& Ayache, 2005) plus the abdomen Computed Tomography (CT) images (Shen \& Kassim, 2007). These techniques equally entail the demerits of having increased computational expenses, being employed in certain images types, as well as not being able to multitask while processing objects (Shen \& Kassim, 2007; Tauber et al., 2005). In the present paper, we suggested and proposed $\alpha$-Shape theory to employ in the extraction of the initial contour later refined by engaging a conventional active contour model.

\section{Improved Contour Initialization Algorithm}

In diverse situations, the contours of the concerned areas exhibit edge aspects, in addition to the existance of noise, for instance burr noise and Interferential points. Therefore, if the technique is capable of prevailing over the noise, setting of the initial contour can be done next to the ideal contour. In the present paper, we initially engage multi-random initialization founded on active counter to acquire edge points in the concerned areas. Subsequently, the tasks entailed progression of the technique using $\alpha$-Shape supposed to prevent the setback of noise space and multi-objects, while eventually employing $\alpha$-Shape point as the primary seed point for the primary contour to be employed in snake technique (Active Contour). Figure 5 has offered a summary of the proposed algorithm flowchart. 


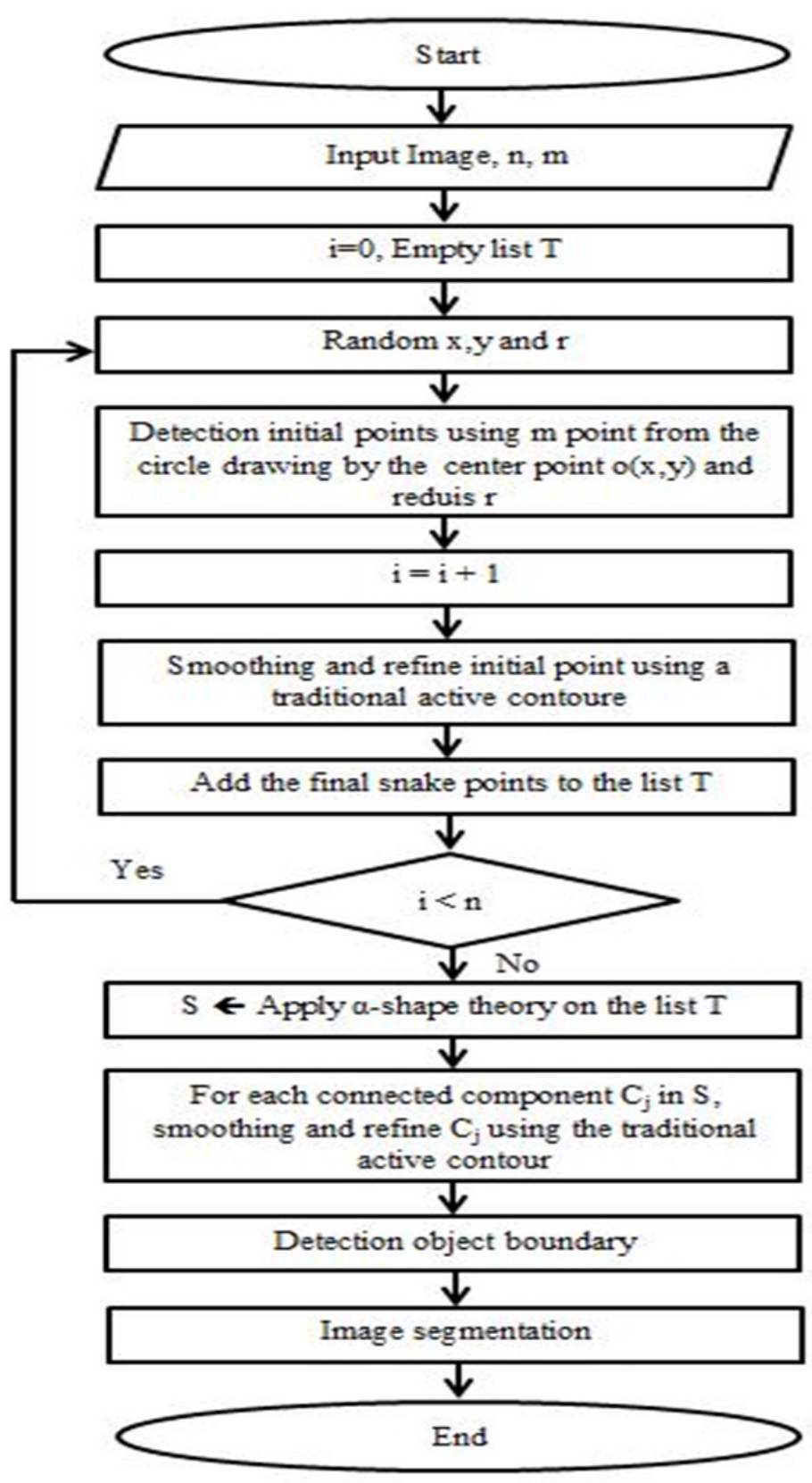

Figure 5. Flowchart of the proposed hybrid algorithm method

\subsection{Multi Random Initialization Based on Active Contour}

Since the adapted active contour segmentation technique is founded on curve development, we employ a circle as the first curve whose radius and center require random setting. Acquisition of edge points of concerned areas is the primary approach in the suggested method. We engage multi-random commencement founded on an active contour to detect and get rid of this point. For this phase, we identify the active contour algorithm "n" time (in the present study $n=10$ ) by engaging three random values $x, y$, and $r$ in each time, to denote "Horizontal Coordinate" and "Vertical Coordinate" of the circle center and circle radius in that order. By engaging $\mathrm{N}$ first contours in an active contour replica, we obtained an $\mathrm{N}$ point list to denote " $\mathrm{n}$ " snakes as the edge points of concerned areas. Table 4 below denotes five-colour images availed in the outcome of this phase, while Algorithm 1 illustrates the pseudo code of this phase. 
Table 5. Result of multi random initialization based on active contour

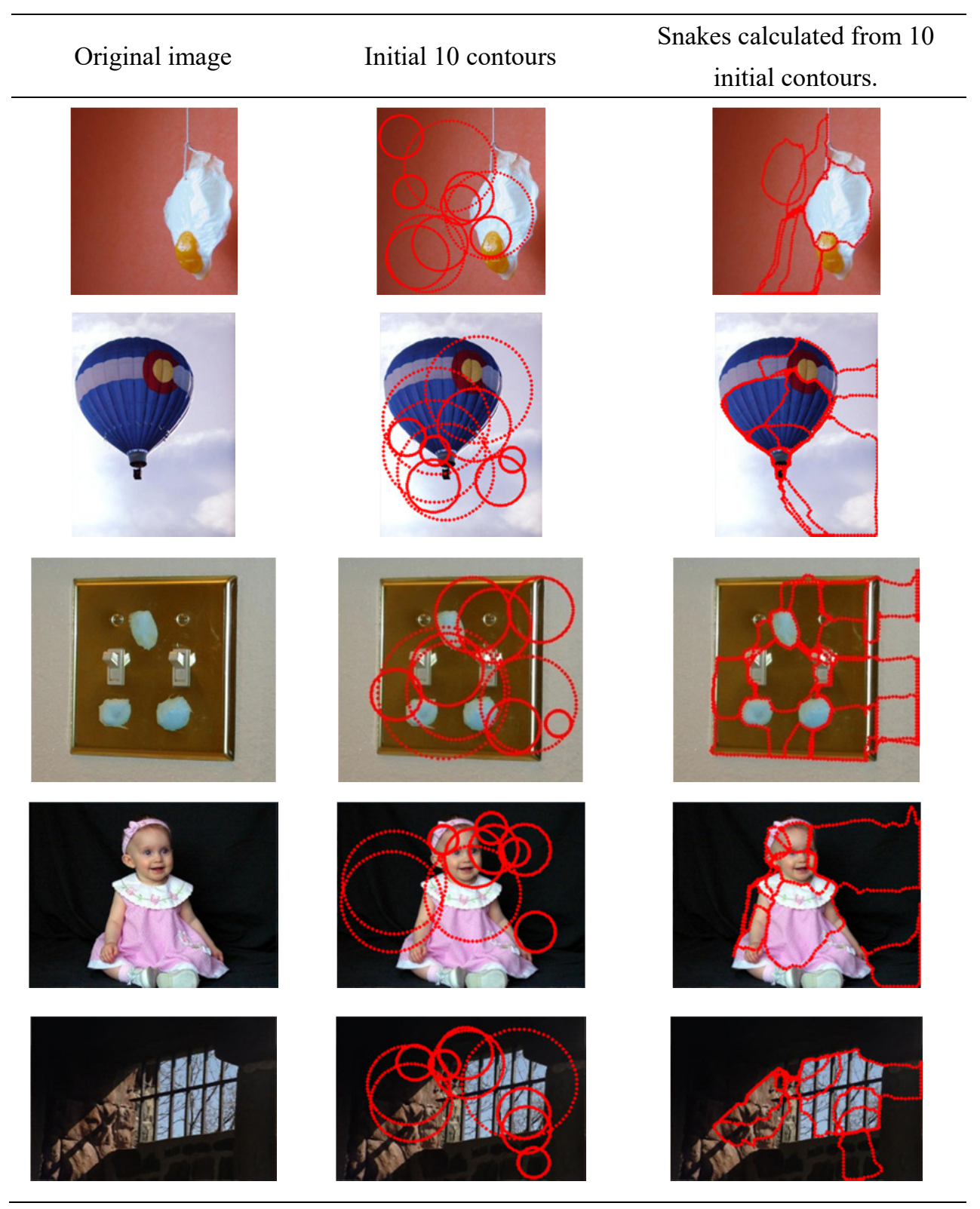




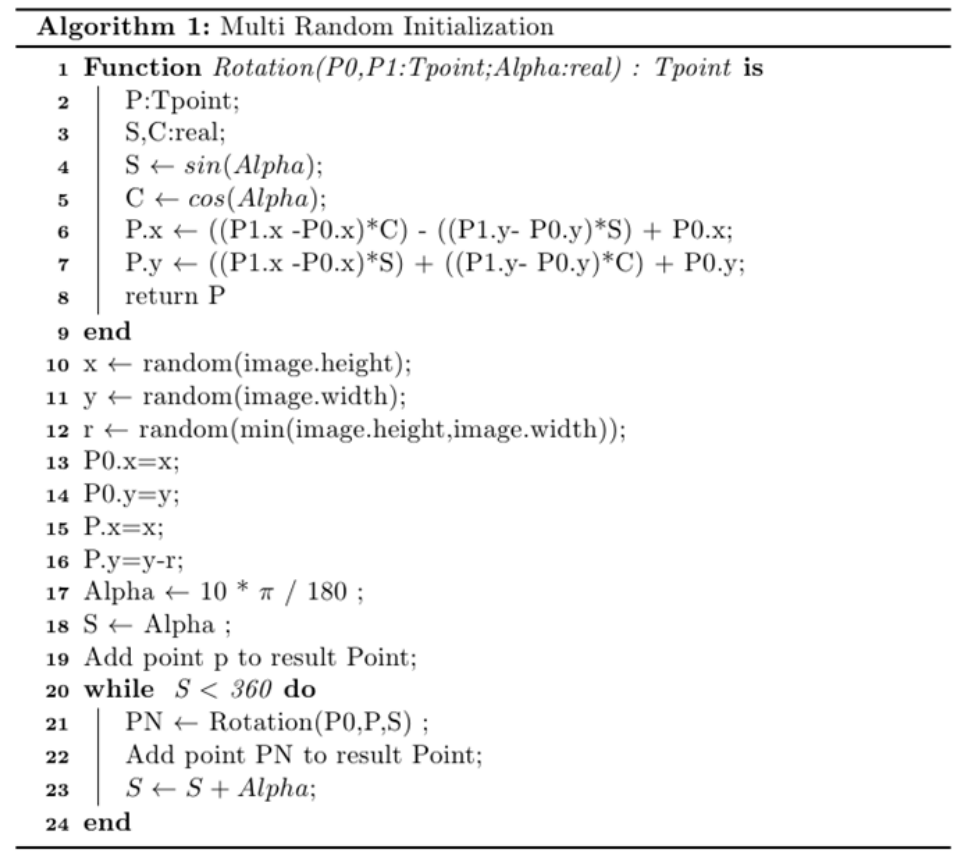

\subsection{Alpha Shape ( $\alpha$-Shape)}

$\alpha$-Shape (commonly identified as Alpha Shape), is a class of piecewise linear plain curves in the Euclidean Plane linked with the shape of a limited points set within computational geometry (Al-Tamimi, Sulong, \& Lut, 2015). Edelsbrunner, Kirkpatrick and Seidel (H. Edelsbrunner, Kirkpatrick, \& Seidel, 1983; Herbert Edelsbrunner, 2010) were the first scholars to define $\alpha$-Shape. The present study discusses $\alpha$-Shape in a detailed approach as they are the study's basic tool for the hybrid algorithm. $\alpha$-Shape have not been overtly employed in motion planning, regardless of being highly utilized in chemistry, biology and geometry as a universal purpose topological and geometric modeling tool. The concept of $\alpha$-Shape is an officiated approach of the instinctive perception of "shape" for spatial point sets. This is arguably contrary to other techniques, just like in surfaces and obtainable surface area, typically estimating and reliant upon several meagerly delineated variables.

Hitherto, $\alpha$-Shape has been engaged in several varied fields. The apparition of associations among data points in both Three Dimensional (3D) and Two Dimensional (2D) is frequently an initial phase of statistical implication. $\alpha$-Shape have been utilized in the visualization of asymmetrical shape margins of 3D clusters (Lucieer \& Kraak, 2004). Further scholars (Moran \& Wagner, 1994) engaged $\alpha$-Shape in characterizing and envisaging a number of simple Brownian motion paths qualities and realized that $\alpha$-Shape are very efficient tools used in gauging a diffusing particle's mass. Arguably, a typical use of $\alpha$-Shape as delineated by several researchers is as a method for enhancing surface renovation from finitely trialed points (Giesen, Cazals, Pauly, \& Zomorodian, 2006; Guo, Menon, \& Willette, 1997; Park, Lee, \& Kim, 2005; Teichmann \& Capps, 1998). Within the area of solid mechanics, $\alpha$-Shape have been engaged in enhancing the interpolation of surface via preventing linear disarticulation fields along convex margins (Teichmann \& Capps, 1998).

$\alpha$-Shape equally offer highly precise linear interpolation over non-convex margins (Cueto, Doblare, \& Gracia, 2000). Lately, within the field of image segmentation, boundaries reconstruction from non-optimal or noisy image segmentation has been efficient, courtesy of $\alpha$-Shape (Meine, Köthe, \& Stelldinger, 2009). By considering trials within atomic levels, Zomorodian and colleagues (Zomorodian, Guibas, \& Koehl, 2006) employed $\alpha$-Shape in an attempt to enhance prediction of protein structure with statistical capabilities. These techniques are practically costly owing to huge number of atomic interfaces, as well as $\alpha$-Shape being engaged to filter the list of protein's interrelating atoms (Rohs et al., 2009; Rother, Hildebrand, Goede, Gruening, \& Preissner, 2009; W. Zhou, Yan, \& Hao, 2012; W. Zhou \& Yan, 2010; Weiqiang Zhou \& Yan, 2010, 2014). In addition, $\alpha$-Shape have equally been engaged in studying structures in proteins (Cheng \& Shi, 2009; De-Alarcón, Pascual-Montano, Gupta, \& Carazo, 2002; Pérot, Sperandio, Miteva, Camproux, \& Villoutreix, 2010; Rohs et al., 2009; Wilson, Bender, Kaya, \& Clemons, 2009), surface area and volume (J Liang, Edelsbrunner, Fu, Sudhakar, \& Subramaniam, 1998; Lou, Jiang, Scott, \& Paul, 2013; Nikkilä, Polishchuk, \& Krasnoshchekov, 2014; Pesaresi \& Schwingshackl, 2014), pockets (H Edelsbrunner, Facello, \& Liang, 1998; J Liang et al., 1998), as well as 
packing (J Liang \& Dill, 2001). With reference to existing literature characterizing voids, irregular pockets, and depressions (J Liang \& Dill, 2001), Liang and colleagues (W. Zhou \& Yan, 2010) assessed the idea of protein packing. They realized a huge resemblance between packed spheres and proteins as compared to that of jig-saw puzzle. They equally found out that protein voids and cavities highly contributed to non-homogenous densities. In addition, by considering proteins of different sizes, the researchers found out how larger proteins are less dense compared to smaller proteins. Therefore, the $\alpha$-Shape affiliated with several points is a simplification of the notion of the convex hull, that is, each of the convex hulls is a $\alpha$-Shape, but not every $\alpha$-Shape is a convex hull

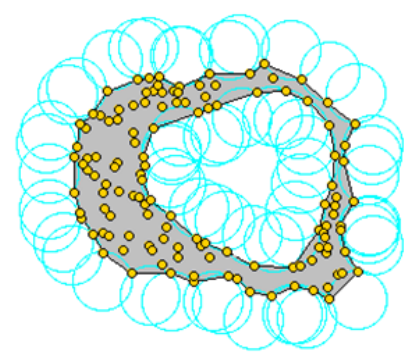

Figure 6. $\alpha$-Shape representation for set of points (Al-Tamimi et al., 2015)

By taking into assumption that provision of 2D points $\mathrm{S}$ set is done, there is the need to have an outcome such as "the shape created by these points." By itself, this idea is highly vague with a probability of several likely interpretations, with $\alpha$-Shape being amongst them. $\alpha$-Shape are capable of being utilized in reconstructing shapes by engaging a dense unorganized dataset points. Actually, an $\alpha$-Shape is segregated by a frontier, identified as a linear estimation of the inventive shape (Bernardini \& Bajaj, 1997).

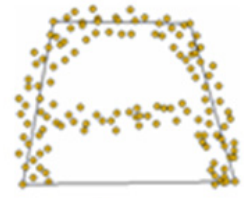

a

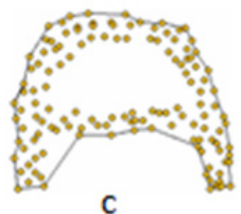

C

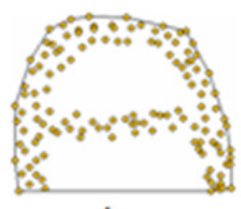

b

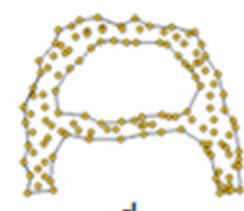

d

Figure 7. Identical set of point and four different frontiers, (a) Linear frontier, (b) Convex hull, (c) Concave hull, and (d) $\alpha$-Shape (Al-Tamimi et al., 2015)

As stipulated in a study by Edelsbrunner (Herbert Edelsbrunner \& Mucke, 1994), the approach delineates a category of shapes, known as $\alpha$-Shape for any provided point set. It enhances accurate, swift and efficient volume and surface area computation.

- $\quad \alpha$-Shape are simplification of the convex hull. Taking into consideration a set point, S.

- For $\alpha=$ infinity, then, $\alpha$-Shape is convex to hull of $\mathrm{S}$.

- In case $\alpha$ is lesser than half the smallest distance amid two points in S, the $\alpha$-Shape is $\mathrm{S}$ itself.

For any $\alpha$ in the middle, it would be perceived that $\alpha$-hull is the largest polygon (polyhedron) or equally a set with vertices being in point set while edges entail a length not beyond $2 \alpha$. Edge presence is indicative of a radius; $\alpha$ probe is not able to pass between the endpoints of the edges. 

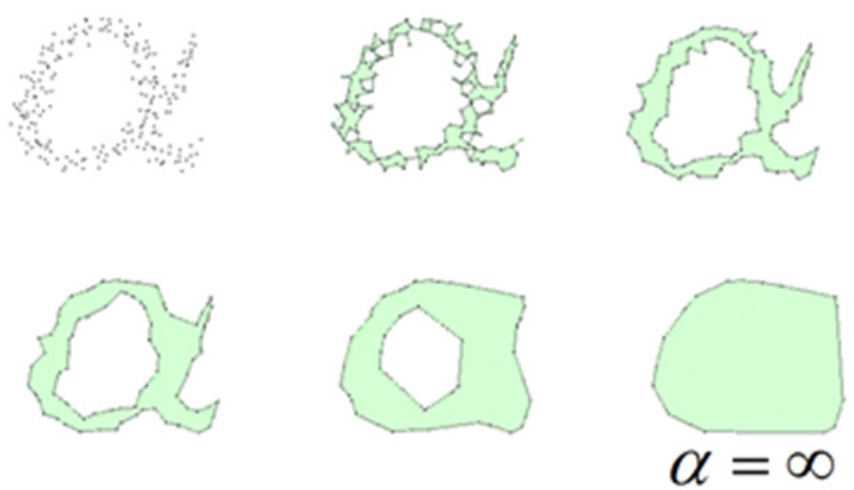

Figure 8. $\alpha$ control the desired level of detail in $\alpha$-Shape theory (Al-Tamimi et al., 2015)

\subsection{Computing the $\alpha$-Shape Using Delaunay Triangulation}

In computational geometry, $\alpha$-Shape computation is arguably the most aptly assessed problems based on Delaunay Triangulation (DT) (O'rourke \& Joseph, 1994). There is a DT for each point sets in 2D. It is highly unique provided co-circular is not met in the four points set, since it lessens the small angles and circumscribed circles. To have the algorithm implemented, there the need to consider the functions below, the primary one is to locate the closest point, as delineated in Algorithm 2.

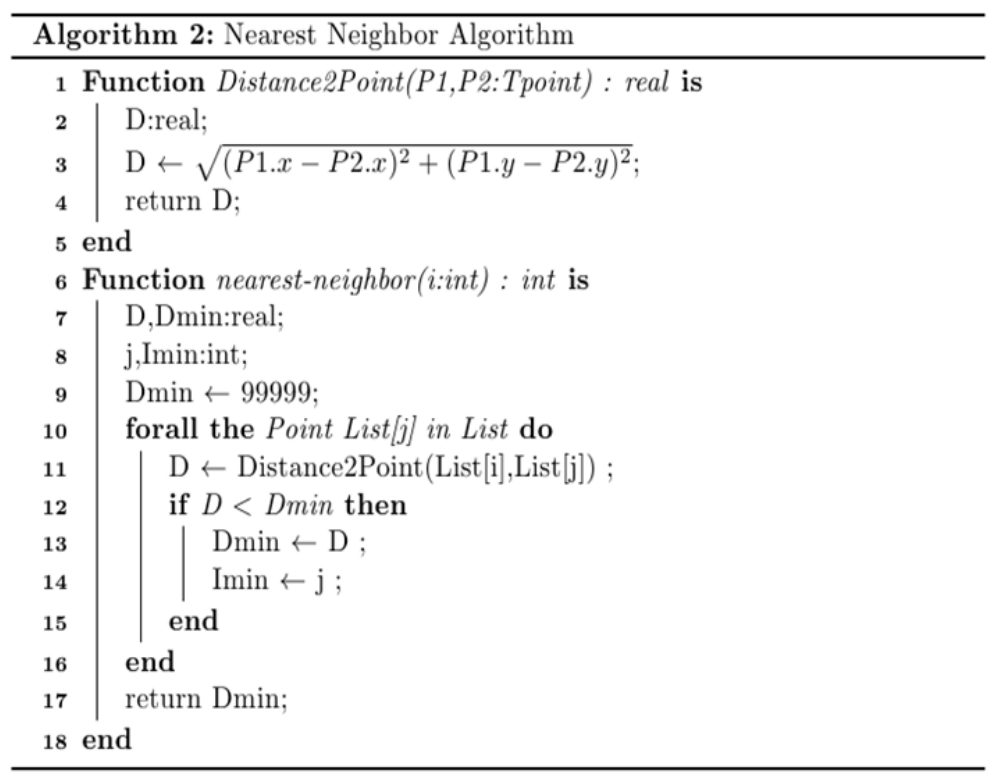

Algorithm 3 shown above return the point vsd such as the triangle, $\mathrm{j}$, vsd is DT, and the point vsd is en right of the line $\mathrm{ij}$, the algorithm return (-1) if we don't find this point. 


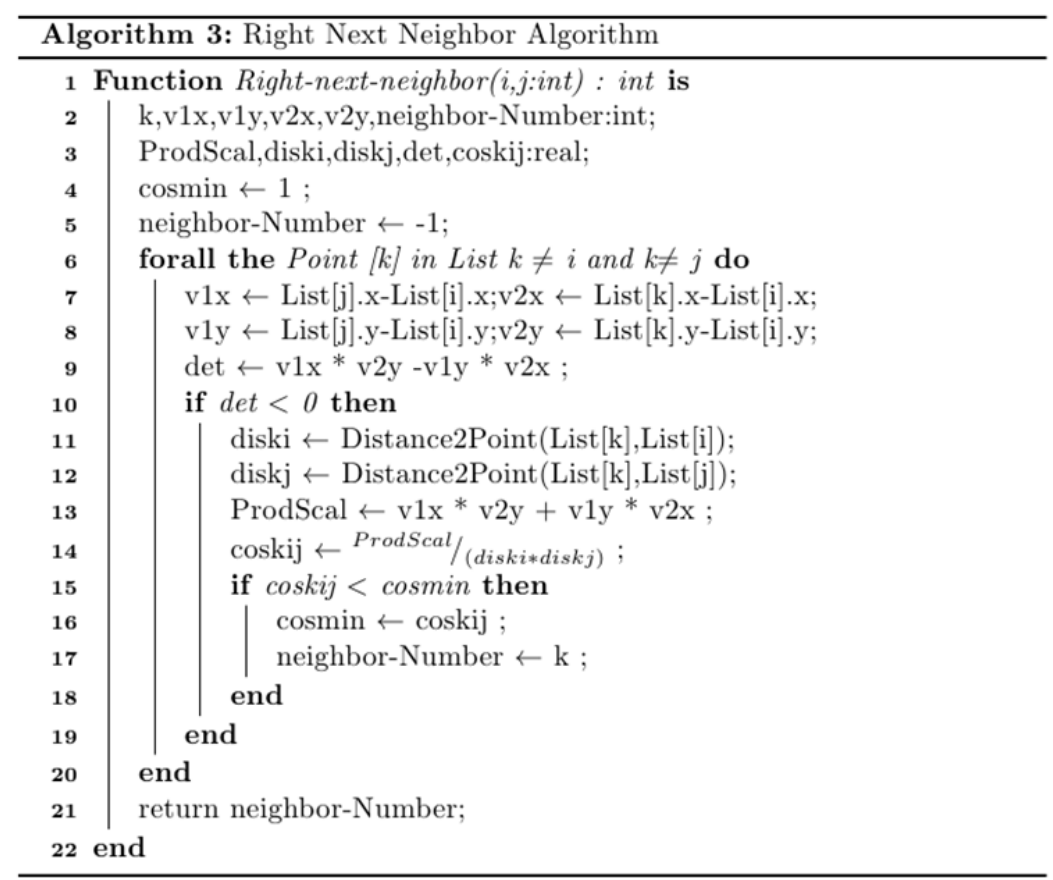

The same algorithm we need to implement to find the point vsg such as the triangle $i, j$, vsg is DT, and the point vsg is en left of the line ij as shown in Algorithm 4:

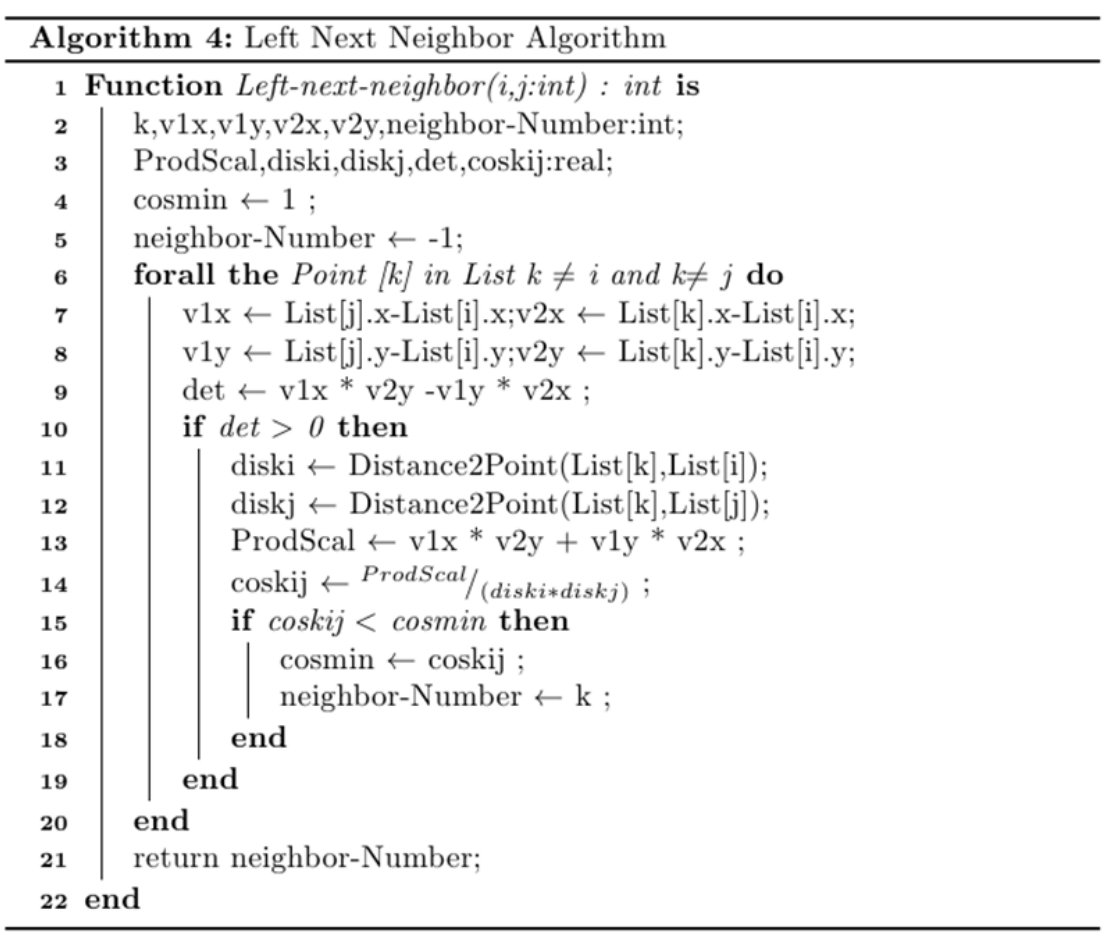

Algorithm 5 shown down resume all the steps need to calculate the DT: 


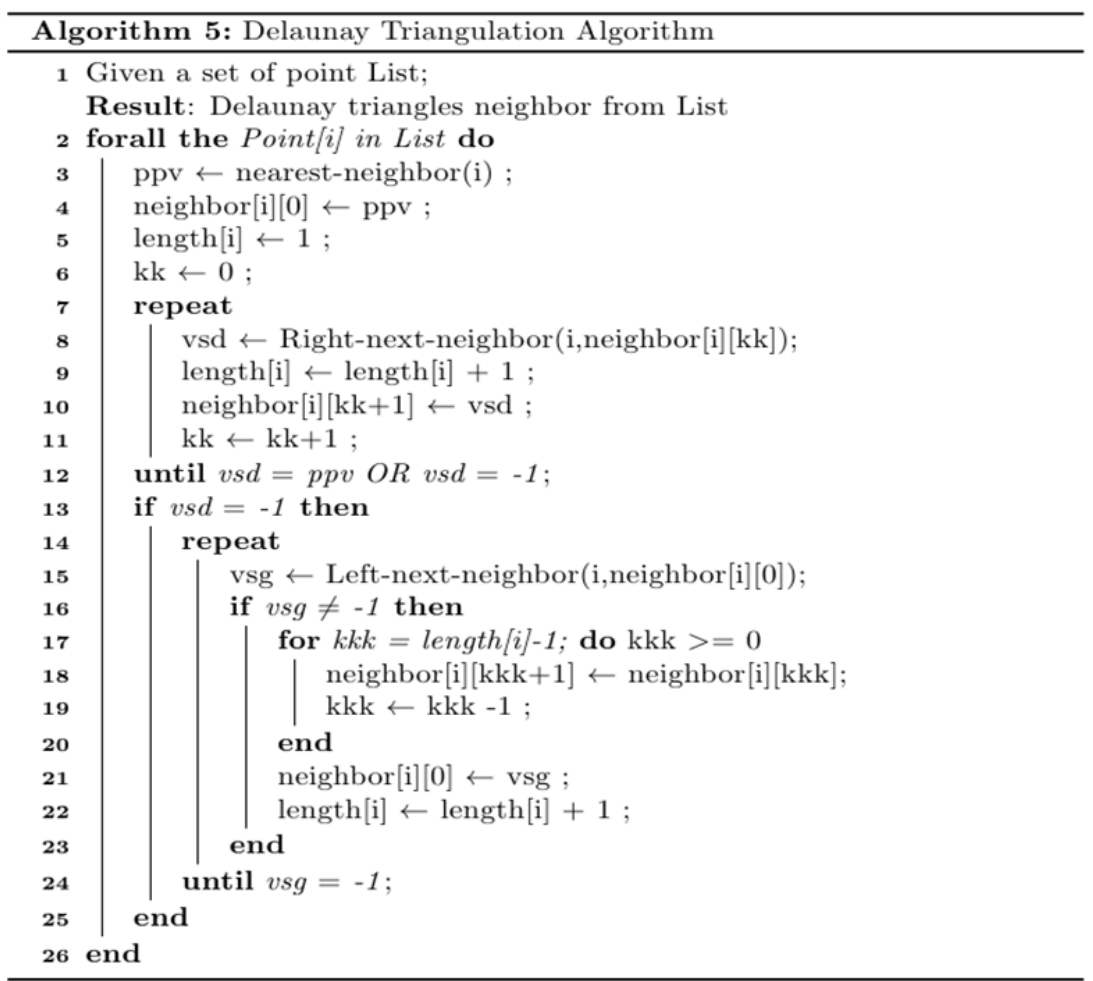

With a point set S provided, DT (S) becomes the DT in a way that no S point is contained in the circumsphere of any d-simplexes $\sigma_{T}$ with $\mathrm{T} \subseteq \mathrm{S}$. The association between $\alpha$-Shape and DT is that $\alpha$-Shape S boundary and DT subset of $\mathrm{S}$, typically identified as $\alpha \mathrm{S} \subseteq \mathrm{DT}(\mathrm{S})$. The following are the key procedures to follow while constructing the $\alpha$-Shape of S: (1) Construct DT, (2) Determine the $\alpha$-extreme points of S, (3) Determine the $\alpha$-neighbors of S, (4) Output the $\alpha$-Shape.

Algorithm 6 delineates the shape computation engaging DT, and Table 5 portrays the $\alpha$-Shape algorithm used on diverse colour images using the set of point calculated by step one of our algorithm.

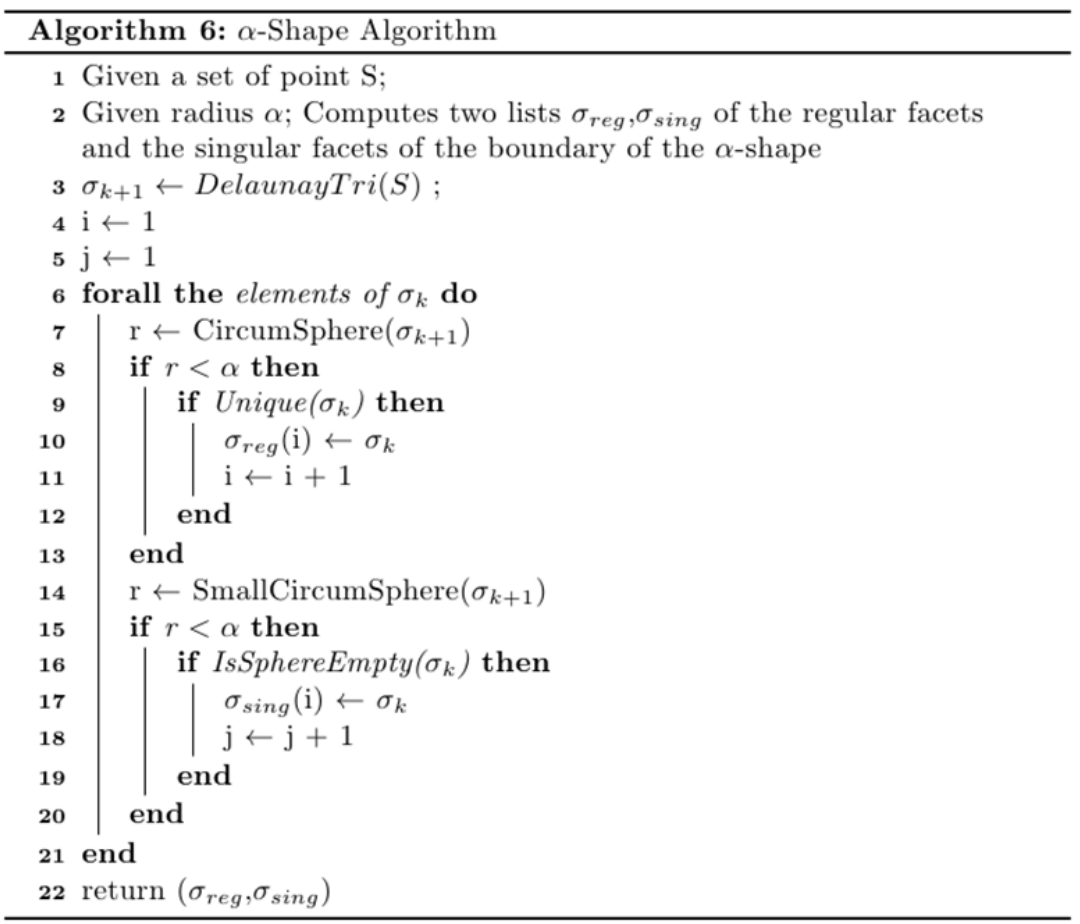


Table 6. $\alpha$-Shape algorithm applied on different colour images using the set of point calculated by step of our algorithm (Continue ...)
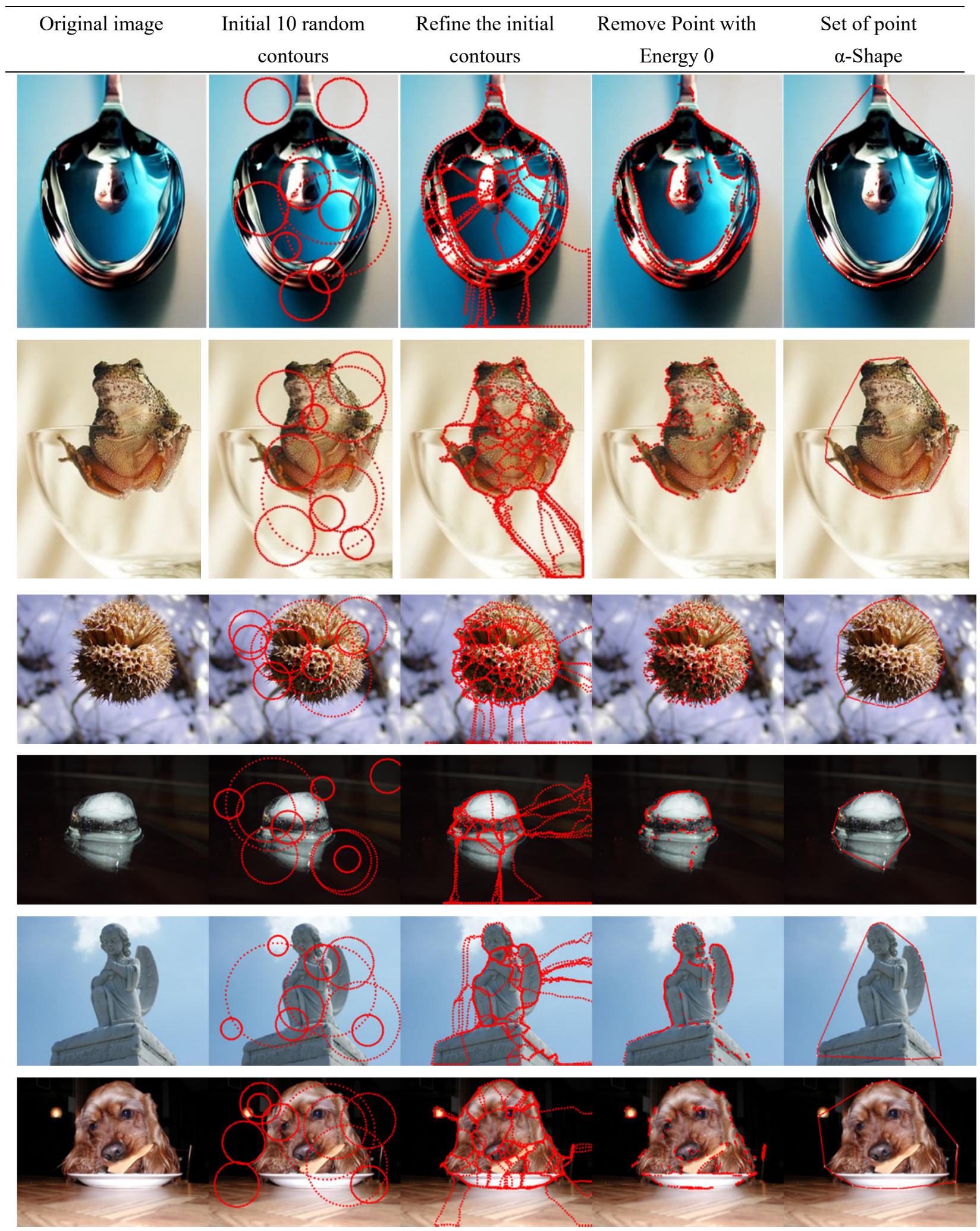
Table 7. $\alpha$-Shape algorithm applied on different colour images using the set of point calculated by step of our algorithm
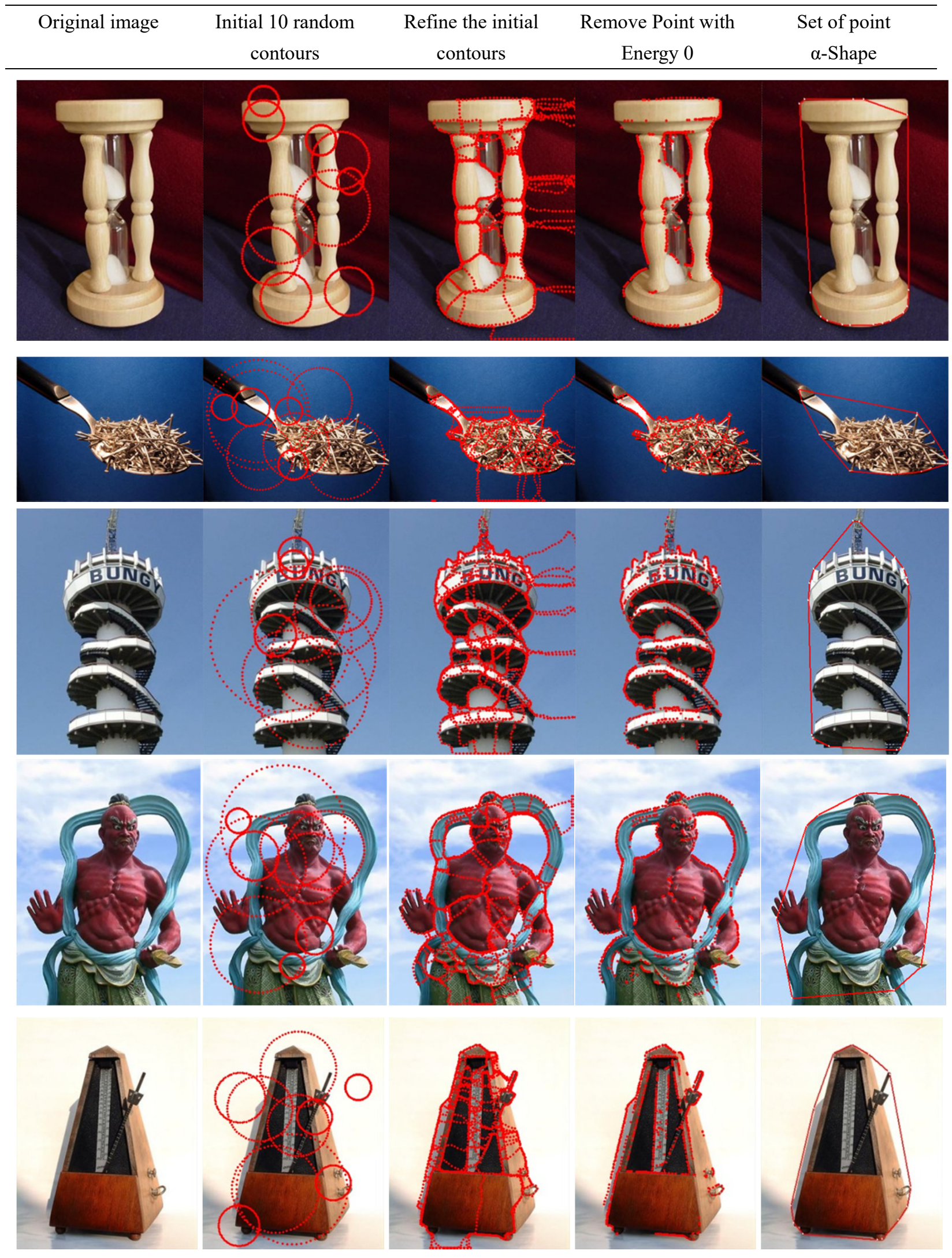
By considering Table 5, it is evident that the $\alpha$-Shape algorithm enhanced the initial contour and facilitated the key point to be engaged in the initialization algorithm of active contour. Furthermore, additional benefits of engaging $\alpha$-Shape cab are evident in case more than a single object is present in the image, employing the merged component algorithm, this has been deeply delineated in the subsequent section.

\subsection{Hybrid Algorithm}

Upon obtaining the $\alpha$-Shape contour, there is the need to have the contour refined to enable it the nearly entire segmentation engaged as template contour image segmentation. There is the need to identify the perfusion location in the opted image. Employing the attached object algorithm (Algorithm 7) is capable of detecting multi-object in identical picture, with demarcation of $\alpha$-shape being done by a frontier of every isolated object. Eventually, for every identified object in the preceding step, each isolated object's frontier is set as the initial contour later refined by engaging the active contour algorithm, employed as template contour image segmentation.

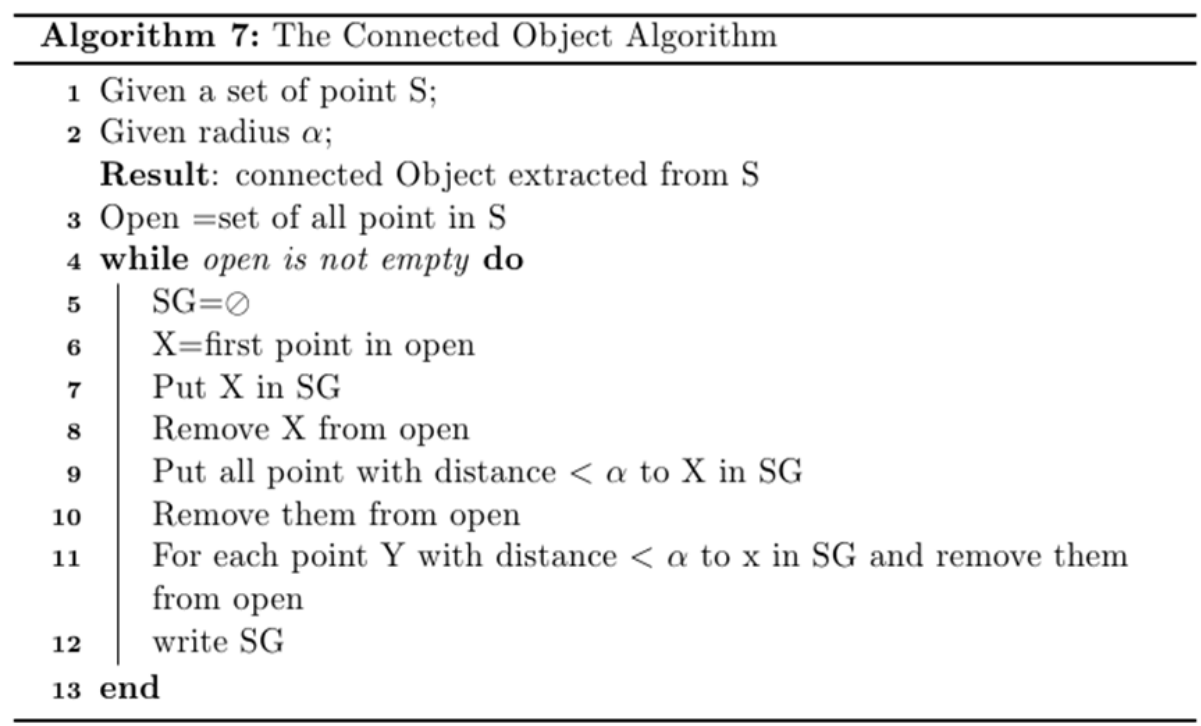

\section{Result and Discussion}

This section offers the likelihood of the use of the suggested hybrid approaches on ideal images. The trial was conducted in Delphi 7.0 by engaging prevailing natural images accessible at the segmentation evaluation database (Alpert, Galun, Brandt, \& Basri, 2012), we conducted our algorithm for the images and assessed the suggest approach. The visual assessment done for the data set segmentation was excellent and offered positive outcome. Table 6 below shows the outcome of segmentation concerning 32 images.

Table 8. Segmentation Results of 32 Randomly Selected Images with one and two objects (Continue ...)

\begin{tabular}{cccc}
\hline RGB image & Segmentation & RGB image & Segmentation \\
\hline 0 & & & \\
\hline
\end{tabular}


Table 9. Segmentation Results of 32 Randomly Selected Images with one and two objects (Continue ...)

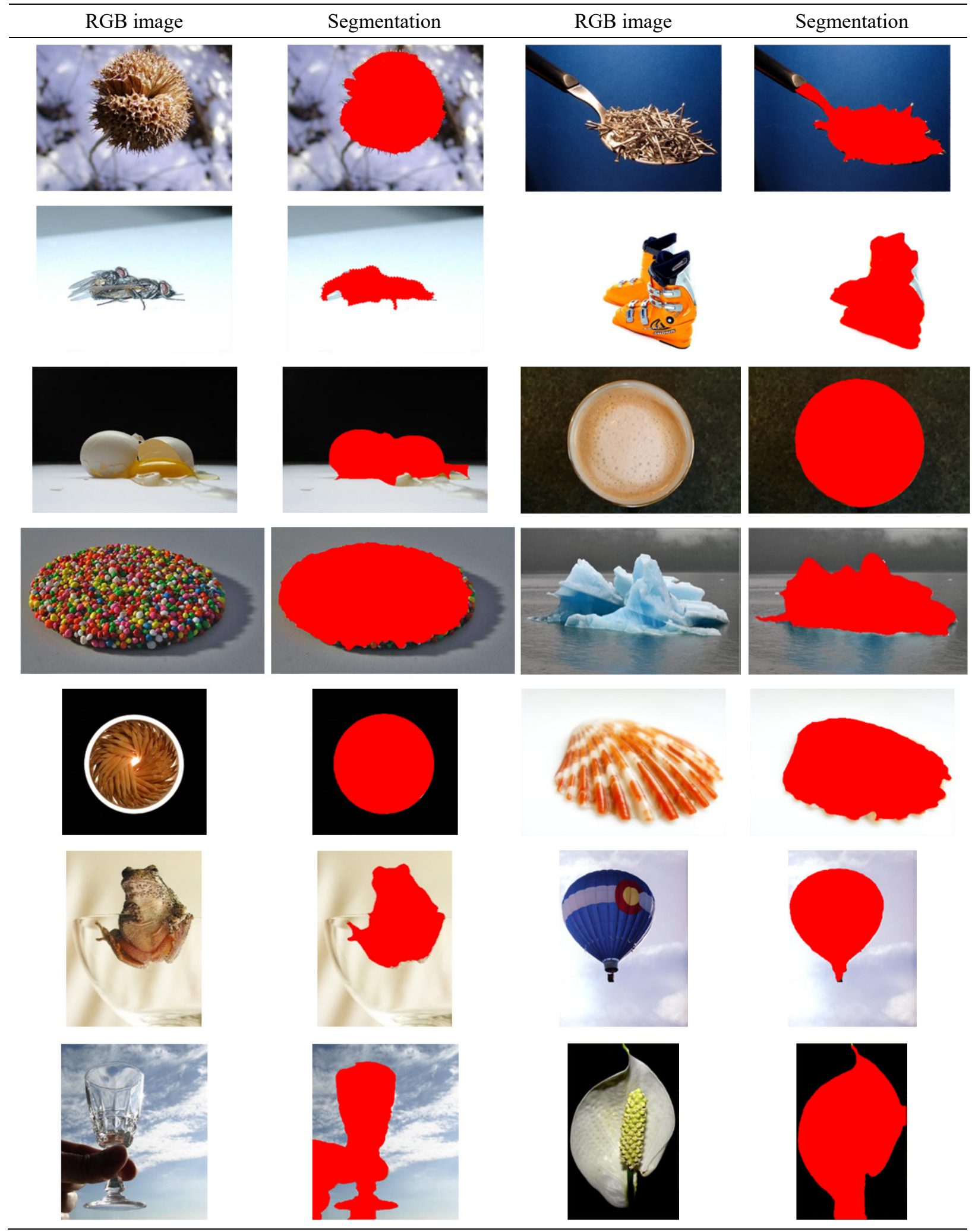


Table 10. Segmentation Results of 32 Randomly Selected Images with one and two objects (Continue ...)

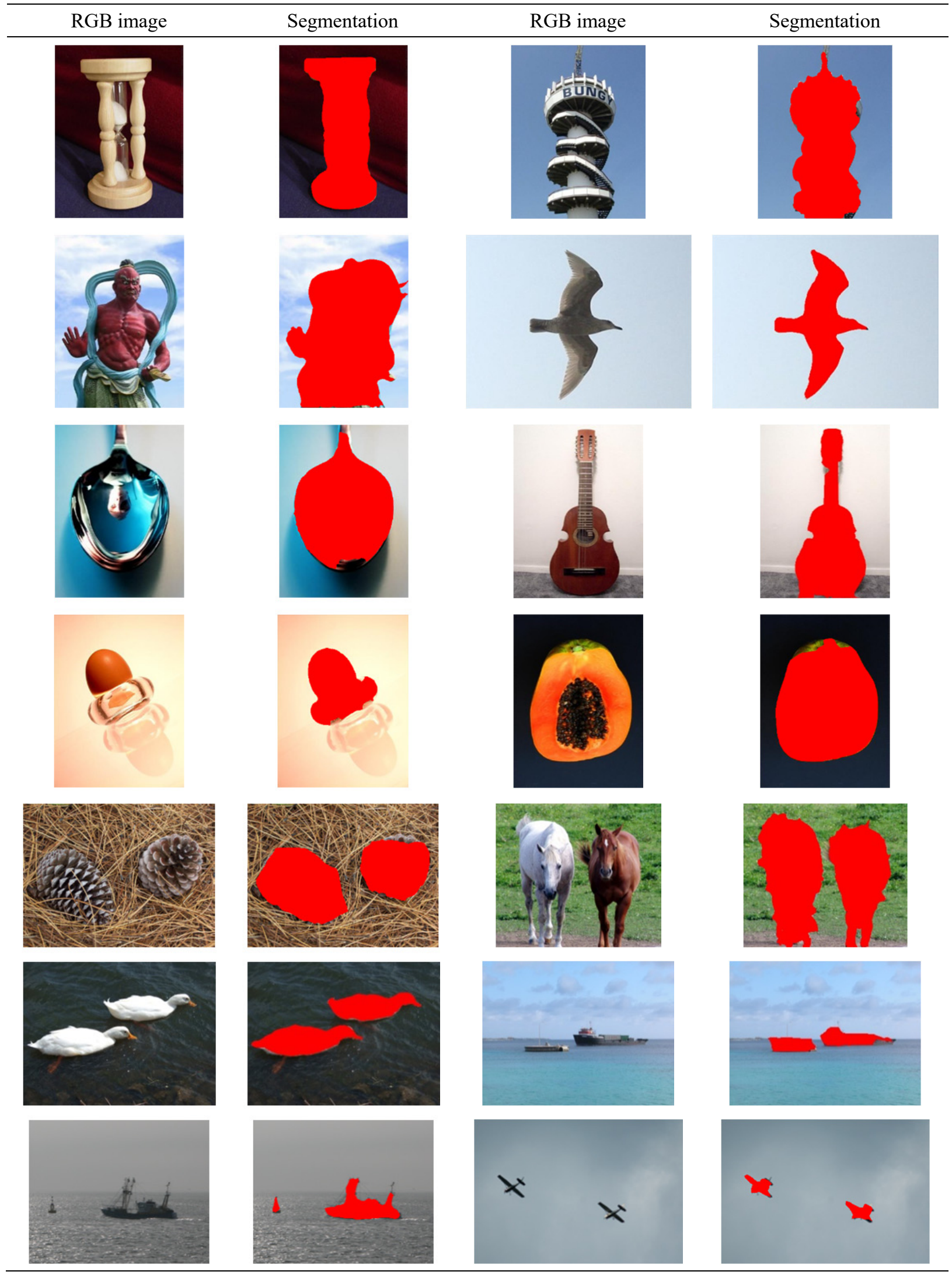


Table 11. Segmentation Results of 32 Randomly Selected Images with one and two objects

RGB image $\quad$ Segmentation $\quad$ RGB image Segmentation

For the outcome, the edge term never highly impacted the image segmentation. The technique could engage textual image statistics instead of conjunction with intensity statistics. Nonetheless, integrating intricate region statistics is capable of exhibiting negative influence on the general algorithm's computational cost.

\section{Conclusions}

This paper has introduced and discussed a hybrid technique for image segmentation founded on $\alpha$-Shape and active contour model. With reference to the mean curvature motion of the guardian vector flow and edge attraction term, the hybrid approach precisely and effectively segmented the natural images. The outcome of the study was highly promising. The type of statistics that could be integrated in the algorithm's region portion as well as the litheness of the parameter setting's approach could highly consider images segmentation with sharp intensity ingredients within areas of interest or weak edges in intensity homogeneity regions. The study proposes that in future there should be an experiment on other area statistics for segmentation and enhances the approach to multispectral images.

\section{Acknowledgements}

The author would like to thank the Ministry of Higher Education \& Scientific Research-Iraq and University of Baghdad for providing the financial support and facilities for this research.

\section{References}

Alpert, S., Galun, M., Brandt, A., \& Basri, R. (2012). Image Segmentation by Probabilistic Bottom-Up Aggregation and Cue Integration. IEEE Transactions on Pattern Analysis and Machine Intelligence, 34(2), 315-326. http://dx.doi.org/10.1109/TPAMI.2011.130

Al-Tamimi, M. S. H., \& Sulong, G. (2014). A Review of Snake Models in Medical MR Image Segmentation. Jurnal Teknologi, 2(1), 101-106.

Al-Tamimi, M. S. H., Sulong, G., \& Lut, I. (2015). Alpha Shape Theory for 3D Visualization and Volumetric Measurement of Brain Tumor Progression Using Magnetic Resonance Images. Magnetic Resonance Imaging, 33, 787-803. http://dx.doi.org/10.1016/j.mri.2015.03.008

Bernardini, F., \& Bajaj, C. L. (1997). Sampling and Reconstructing Manifolds Using Alpha-Shapes. Purdue E-Pubs, a Service of the Purdue University Libraries, 1-11.

Caixia, L., Yanbin, F., \& Houjun, Y. (2006). A New Algorithm of Contour Initialization in GVF Snake Model. Computer Application, 26(7), 1614-1616.

Cheng, H. L., \& Shi, X. (2009). Quality Mesh Generation for Molecular Skin Surfaces Using Restricted Union of Balls. Computational Geometry, 42(3), 196-206. http://dx.doi.org/10.1016/j.comgeo.2008.10.001

Choi, W., Lam, K., Siu, W., \& Ic, Q. L. (2001). An Adaptive Active Contour Model for Highly Irregular Boundaries. Pattern Recognition Society, 34(2), 323-331.

Codon, P. D. P. Y., \& Fristot, V. (1999). Automatic Snakes for Robust Lip Boundaries Extraction. In In Acoustics, Speech, and Signal Processing, 1999. Proceedings., 1999 IEEE International Conference (Vol. 6, pp. 3069 3072).

Cueto, E., Doblare, M., \& Gracia, L. (2000). Imposing Essential Boundary Conditions in the Natural Element Method by Means of Density-Scaled Alpha-Shapes. International Journal for Numerical Methods in Engineering, 49(4), 519-546. 
De-Alarcón, P. A, Pascual-Montano, A., Gupta, A., \& Carazo, J. M. (2002). Modeling Shape and Topology of Low-Resolution Density Maps of Biological Macromolecules. Biophysical Journal, 83(2), 619-632. http://dx.doi.org/10.1016/S0006-3495(02)75196-5

Edelsbrunner, H. (2010). Alpha Shapes - a Survey. Tessellations in the Sciences, 1-25.

Edelsbrunner, H., Facello, M., \& Liang, J. (1998). On the Definition and the Construction of Pockets in Macromolecules. Discrete Applied Mathematics, 88(1), 83-102. Retrieved from http://www.ncbi.nlm.nih.gov/pubmed/9390238

Edelsbrunner, H., Kirkpatrick, D., \& Seidel, R. (1983). On the Shape of a Set of Points in the Plane. IEEE Transactions on Information Theory, 29(4), 551-559. http://dx.doi.org/10.1109/TIT.1983.1056714

Edelsbrunner, H., \& Mucke, E. P. (1994). Three-Dimensional Alpha Shapes. ACM Transactions on Graphics (TOG), 13(1), 43-72.

Gastaud, M., Fellow, M. B., Aubert, G., \& Ý, Ü. Ý. Ü. (2004). Combining Shape Prior and Statistical Features For Active Contour Segmentation. Circuits and Systems for Video Technology, IEEE Transactions, 2-10.

Giesen, J., Cazals, F., Pauly, M., \& Zomorodian, A. (2006). The Conformal Alpha Shape Filtration. The Visual Computer, 22(8), 531-540. http://dx.doi.org/10.1007/s00371-006-0027-1

Guo, B., Menon, J., \& Willette, B. (1997). Surface Reconstruction Using Alpha Shapes. Computer Graphics Forum, 16(4), 177-190. http://dx.doi.org/10.1111/1467-8659.00178

Haud, J.-O. L., \& Montanvert, A. (1999). Deformable Meshes With Automated Topology Changes for Coarse-to-Fine Three - Dimensional Surface Extraction. Med. Image Anal., 3, 187-207.

Kass, M., Witkin, A., \& Terzopoulos, D. (1987). Snakes: Active Contour Models. International Journal of Computer Vision, 1(14), 321-331.

Liang, J., \& Dill, K. A. (2001). Are Proteins Well-Packed? Biophysical Journal, 81(2), 751-766. http://dx.doi.org/10.1016/S0006-3495(01)75739-6

Liang, J., Ding, G., \& Wu, Y. (2008). Segmentation of the Left Ventricle from Cardiac MR Images Based on Radial GVF Snake. In In BioMedical Engineering and Informatics, 2008. BMEI 2008. International Conference (Vol. 2, pp. 238-242). IEEE. http://dx.doi.org/10.1109/BMEI.2008.188

Liang, J., Edelsbrunner, H., Fu, P., Sudhakar, P. V, \& Subramaniam, S. (1998). Analytical Shape Computation of Macromolecules: I. Molecular Area and Volume Through Alpha Shape. Proteins Structure Function and Genetics, 33(1), 1-17. Retrieved from http://www.ncbi.nlm.nih.gov/pubmed/9741840

Lou, S., Jiang, X., Scott, P. J., \& Paul, J. (2013). Application of the Morphological Alpha Shape Method to the Extraction of Topographical Features from Engineering Surfaces. Measurement, 46(2), 1002-1008.

Lucieer, A., \& Kraak, M. (2004). $\alpha$-Shapes for Visualizing Irregular Shaped Class Clusters in 3D. Electronic Imaging 2004. International Society for Optics and Photonics, 201-211.

Mansouri, A., Mukherjee, D. P., Member, S., \& Acton, S. T. (2004). Constraining Active Contour Evolution Via Lie Groups of Transformation. Image Processing, IEEE Transactions, 13(6), 853-863.

McInerney, T., \& Terzopoulos, D. (1999). Topology Adaptive Deformable Surfaces for Medical Image Volume Segmentation. IEEE Transactions on Medical Imaging, 18(10), 840-850. http://dx.doi.org/10.1109/42.811261

Meine, H., Köthe, U., \& Stelldinger, P. (2009). A Topological Sampling Theorem for Robust Boundary Reconstruction and Image Segmentation. Discrete Applied Mathematics, 157(3), 524-541. http://dx.doi.org/10.1016/j.dam.2008.05.031

Moran, P. J., \& Wagner, M. (1994). Introducing Alpha Shapes for the Analysis of Path Integral Monte Carlo Results. In Visualization'94, Proceedings., IEEE Conference on. IEEE (pp. 52-59). IEEE Comput. Soc. Press. http://dx.doi.org/10.1109/VISUAL.1994.346337

Nikkilä, M., Polishchuk, V., \& Krasnoshchekov, D. (2014). Robust Estimation of Seismic Coda Shape. Geophysical Journal International, ggu002, 1-18.

O'rourke, \& Joseph. (1994). Computational Geometry Column 22. International Journal of Computational Geometry \& Applications, 4(01), 119-122.

Ootes, T. F. C., Aylor, C. J. T., Ooper, D. H. C., \& Graham, J. (1995). Active Shape Models-Their Training and 
Application. Computer Vision and Image Understanding, 61(1), 38-59.

Paragios, N., \& Deriche, R. (2000). Geodesic Active Contours and Level Sets for the Detection and Tracking of Moving Objects. Pattern Analysis and Machine Intelligence, IEEE Transactions, 22(3), 266-280. Retrieved from http://ieeexplore.ieee.org/xpls/abs_all.jsp?arnumber=841758\&tag=1

Park, S. H., Lee, S. S., \& Kim, J. H. (2005). A Surface Reconstruction Algorithm Using Weighted Alpha Shapes. In Fuzzy Systems and Knowledge Discovery. Springer Berlin Heidelberg, 1141-1150.

Pérot, S., Sperandio, O., Miteva, M. A., Camproux, A. C., \& Villoutreix, B. O. (2010). Druggable Pockets and Binding Site Centric Chemical Space: a Paradigm Shift in Drug Discovery. Drug Discovery Today, 15(15), 656-667.

Pesaresi, L., \& Schwingshackl, C. W. (2014). Automated Measurement Grid Generation for Scanning Laser Doppler Vibrometers. Topics in Modal Analysis, Springer New York, 7, 645-653.

Pichumani, R. (1997). Survey of Current Techniques. Retrieved from http://homepages.inf.ed.ac.uk/rbf/CVonline/LOCAL_COPIES/RAMANI1/node17.html

Pluempitiwiriyawej, C., \& Sotthivirat, S. (2005). Active Contours With Automatic Initialization for Myocardial Perfusion Analysis. In Conference proceedings : Annual International Conference of the IEEE Engineering in Medicine and Biology Society (Vol. 3, pp. 3332-3335). http://dx.doi.org/10.1109/IEMBS.2005.1617190

Prince, J. L. (1997). Gradient Vector Flow: A New External Force for Snakes. In Computer Vision and Pattern Recognition, 1997. Proceedings., 1997 IEEE Computer Society Conference on. IEEE (pp. 66-71). IEEE Comput. Soc. http://dx.doi.org/10.1109/CVPR.1997.609299

Ray, N., \& Acton, S. T. (2004). Motion Gradient Vector Flow: an External Force for Tracking Rolling Leukocytes With Shape and Size Constrained Active Contours. IEEE Transactions on Medical Imaging, 23(12), 1466-1478. http://dx.doi.org/10.1109/TMI.2004.835603

Ray, N., Acton, S. T., \& Ley, K. (2002). Tracking Leukocytes in Vivo With Shape and Size Constrained Active Contours. IEEE Transactions on Medical Imaging, 21(10), 1222-1235. http://dx.doi.org/10.1109/TMI.2002.806291

Rohs, R., West, S. M., Sosinsky, A., Liu, P., Mann, R. S., \& Honig, B. (2009). The Role of DNA Shape in Protein - DNA Recognition. Nature, 1248-1253.

Rother, K., Hildebrand, P. W., Goede, A., Gruening, B., \& Preissner, R. (2009). Voronoia: Analyzing Packing in Protein Structures. Nucleic Acids Research, 37, D393-D395. http://dx.doi.org/10.1093/nar/gkn769

Shen, W., \& Kassim, A. A. (2007). A New Scheme for Automatic Initialization of Deformable Models. In Image Processing, 2007. ICIP 2007. IEEE International Conference (pp. 289-292).

T. McInerney, \& Terzopoulos, D. (1995). A Dynamic Finite Element Surface Model for Segmentation and Tracking in Multidimensional Medical Images With Application to Cardiac 4D Image Analysis. Comput. Med. Imag. Graph., 19, 69-83.

Tauber, C., Batatia, H., \& Ayache, A. (2005). A General Quais- Automatic Initialization for Snakes: Application to Ultrasound Images. In Image Processing, 2005. ICIP 2005. IEEE International Conference (pp. 9-12).

Teichmann, M., \& Capps, M. (1998). Surface Reconstruction With Anisotropic Density-Scaled Alpha Shapes. Visualization'98. Proceedings, 67-72. http://dx.doi.org/10.1109/VISUAL.1998.745286

Terzopoulos, D., Witkin, A., \& Kass, M. (1988). Constraints on Deformable Models: Recovering 3D Shape and Nonrigid Motion. Artificial Intelligence, 36(1), 91-123.

Wilson, J. A., Bender, A., Kaya, T., \& Clemons, P. A. (2009). Alpha Shapes Applied to Molecular Shape Characterization Exhibit Novel Properties Compared to Established Shape Descriptors. Journal of Chemical Information and Modeling, 49(10), 2231-2241. http://dx.doi.org/10.1021/ci900190z

Xu, C., \& Prince, J. L. (1998). Snakes, Shapes, and Gradient Vector Flow. IEEE Transactions on Image Processing: A Publication of the IEEE Signal Processing Society, 7(3), 359-369. $\mathrm{http}: / / \mathrm{dx}$.doi.org/10.1109/83.661186

Xu, C., \& Prince, J. L. (2006). Active Contours, Deformable Models, and Gradient Vector Flow. Available on: Http://iacl. Ece. Jhu. Edu/projects/gvf/via the Internet.

Zhou, W., \& Yan, H. (2010). Prediction of DNA-Binding Protein Based on Alpha Shape Modeling. In 2010 IEEE International Conference on Bioinformatics and Biomedicine (BIBM) (pp. 23-28). IEEE. 
http://dx.doi.org/10.1109/BIBM.2010.5706529

Zhou, W., \& Yan, H. (2010). Relationship Between Periodic Dinucleotides and the Nucleosome Structure Revealed by Alpha Shape Modeling. Chemical Physics Letters, 489(4), 225-228.

Zhou, W., \& Yan, H. (2014). Alpha Shape and Delaunay Triangulation in Studies of Protein-Related Interactions. Briefings in Bioinformatics, 15(1), 54-64. http://dx.doi.org/10.1093/bib/bbs077

Zhou, W., Yan, H., \& Hao, Q. (2012). Analysis of Surface Structures of Hydrogen Bonding in Protein - Ligand Interactions Using the Alpha Shape Model. Chemical Physics Letters, 545, 125-131.

Zomorodian, A., Guibas, L., \& Koehl, P. (2006). Geometric Filtering of Pairwise Atomic Interactions Applied to the Design of Efficient Statistical Potentials. Computer Aided Geometric Design, 23(6), 531-544.

\section{Copyrights}

Copyright for this article is retained by the author(s), with first publication rights granted to the journal.

This is an open-access article distributed under the terms and conditions of the Creative Commons Attribution license (http://creativecommons.org/licenses/by/3.0/). 